\title{
Aplicação do Mindfulness em um Sistema Tutor Inteligente: um Estudo Piloto
}

\author{
Bruno Luis Schaab, Michael Duarte, Otávio Bastos Azevedo, \\ Daniel Viana Abs da Cruz, Patricia A. Jaques
}

Programa Interdisciplinar de Pós-Graduação em Computação Aplicada (PIPCA)
Universidade do Vale do Rio dos Sinos (UNISINOS)
brunoluisschaab@gmail.com, mquadrosduarte@gmail.com,
otaviobastosazevedo@gmail.com, dabs@unisinos.br, pjaques@unisinos.br

\begin{abstract}
This article describes an experimental evaluation with 46 students that sought to improve the attention of learners through mindfulness in an intelligent tutor system (ITS). In the beginning of the sessions, the STI played an audio that guided the experimental group in the practice of mindfulness, while the control group heard a story about algebra. Although no significant evidence of improved performance in tests was found for the experimental group, the instrument of perception showed an improvement on attention in both groups. We believe that listening to an audio or a story about algebra may have been a similar experience to the practice of mindfulness. These results suggest that this research work deserves further investigation.
\end{abstract}

Resumo. Este artigo descreve uma avaliação experimental com 46 alunos que buscou melhorar a atenção dos aprendizes de um sistema tutor inteligente (STI) por meio de mindfulness. No início da sessão, o STI tocava um áudio, que guiava o grupo experimental na prática de mindfulness, enquanto o grupo de controle ouvia sobre a história da álgebra. Embora não há evidências significativas de um melhor desempenho em testes do grupo experimental, o instrumento de percepção de atenção evidenciou uma melhora da atenção em ambos os grupos. Acredita-se que ouvir um áudio sobre a história da álgebra pode ter sido uma experiência parecida à prática de mindfulness. Esses resultados sugerem o interesse de uma continuação do trabalho.

\section{Introdução}

Sistemas tutores inteligentes (STI) são ambientes virtuais de aprendizagem que visam assistir estudantes nos processos de ensino-aprendizagem de determinado domínio [Woolf 2010]. Para tanto, técnicas de inteligência artificial são utilizadas para modelar o conhecimento do estudante e lhe fornecer assistência individualizada. Alguns estudos recentes, envolvendo meta-análise, têm mostrado que STIs podem ser tão efetivos quanto um professor particular [Ma et al. 2014; Steenbergen-Hu e Cooper 2014; Steenbergen-Hu e Cooper 2013]. Em especial, os tutores do tipo step-based, que assistem os estudantes na resolução passo-a-passo de problemas, mostraram-se ainda mais efetivos [Vanlehn 2011].

Por se tratar de um ambiente de aprendizagem, é importante que os usuários se mantenham atentos durante a utilização do programa, uma vez que a atenção está correlacionada positivamente com a aprendizagem [Paula et al. 2006]. Por exemplo, 
crianças que possuem patologias como TDAH (transtorno de déficit de atenção e hiperatividade), em que a atenção se encontra prejudicada, apresentam dificuldades de aprendizagem [Oord et al. 2012]. Deste modo, é possível melhorar a aprendizagem dos estudantes, quando a atenção é favorecida por alguma técnica específica.

Por tal situação, surge a necessidade de algum mecanismo que consiga favorecer a melhora da atenção e também a aprendizagem de um usuário de um STI. Questões relacionadas à atenção têm sido objeto de estudo de diversos psicólogos cognitivos, que lidam com crescentes casos de TDAH, além da falta de atenção e concentração que rotineiramente se encontra em crianças e adolescentes. A prática de meditação, que ganha o nome de mindfulness pelos pesquisadores ocidentais da Psicologia e Neurociência, tem sido empregada como um método eficiente para o ganho de atenção, assim como para a melhora de diversos aspectos do bem-estar. A neurociência, por sua vez, vem estudando as mudanças em estruturas cerebrais em pessoas que realizam mindfulness [Davidson e Lutz 2008].

A prática de mindfulness é um campo de pesquisa promissor na área de aprendizagem em escolas [Flook et al. 2015; Wisner 2013; Felver 2013]. No entanto, não se tem conhecimento de pesquisas relacionadas à mindfulness em sistemas tutores inteligentes ou em ambientes computacionais de aprendizagem não inteligentes. Com o objetivo de melhorar a atenção e, por consequência, a aprendizagem por parte dos alunos, pretende-se integrar a prática de mindfulness a STIs. Na presente versão do trabalho proposto, a prática é guiada por narrações em áudio que são mostradas aos estudantes no início de cada sessão com o sistema tutor. O trabalho proposto foi integrado ao PAT2Math ${ }^{1}$, um web step-based STI que assiste os estudantes na resolução passo-a-passo de equações algébricas.

Foi conduzida uma avaliação experimental, em formato piloto, em duas turmas de alunos do sétimo ano de uma escola particular da região do Vale dos Sinos, no Rio Grande do Sul. Os alunos usaram o STI durante 4 semanas, uma vez por semana, no laboratório da escola. As sessões em laboratório tinham duração de 50 minutos, sendo que 5 minutos (duração da prática) eram destinados ao mindfulness. O estudo se deu com a divisão aleatória de 59 alunos em um grupo de experimento e outro de controle. O primeiro grupo realizou a prática de mindfulness antes da utilização do STI, enquanto o segundo ouviu uma narração acerca da história da álgebra, durante o mesmo período de tempo. Para avaliação de possíveis ganhos de atenção e aprendizagem, uma escala de percepção de atenção foi desenvolvida por estudantes de Psicologia, sob orientação de um psicólogo responsável, além de um teste de álgebra. Não foram encontrados resultados que demonstram a eficiência do mindfulness na aprendizagem. No entanto, o instrumento de percepção de atenção indicou que praticar o mindfulness e ouvir áudio sobre a história da álgebra podem ser experiências similares, apresentando mudanças estaticamente significativas em comportamentos relacionados à atenção.

\section{Mindfulness e sua Inserção no Ambiente Escolar}

O mindfulness é uma forma de atenção plena, isto é, a concentração no momento atual, intencional e sem julgamento [Kabat-Zinn 1990]. Durante a realização de determinada atividade, o praticante deve estar atento para as questões relativas de uma situação

\footnotetext{
${ }^{1}$ http://pat2math.unisinos.br.
} 
específica, sem que o seu foco esteja disperso em pensamentos passados ou futuros, e ainda sem realizar qualquer espécie de julgamento acerca destes pensamentos. Assim sendo, a prática de mindfulness visa apenas que estes pensamentos sejam observados, sem que sejam considerados bons ou ruins [Baer 2003]. A prática pode ser realizada de diversas maneiras: sentado prestando atenção na respiração; caminhando, observando atentamente cada movimento ligado ao ato de caminhar, etc. A forma mais comumente empregada é a observação da respiração, imóvel, numa posição sentada e com coluna ereta. Essa forma de prática tem origem na meditação budista e por isso é também chamada de meditação. Estudos relatam diversos benefícios positivos das técnicas de mindfulness, entre as quais, melhora da atenção, aprendizagem, bem-estar, diminuição dos níveis de ansiedade, entre outros [Weare 2012].

Os resultados de pesquisas que buscam inserir a prática de mindfulness em colégios indicam a melhora de atenção dos alunos participantes, além de outras dimensões importantes na aprendizagem. No estudo descrito em [Flook et al. 2015], 67 estudantes em idade pré-escolar (média $=4,67$ anos), de sete diferentes colégios, participaram de um experimento para avaliar os ganhos comportamentais provenientes da prática de mindfulness. Durante um período de doze semanas, os alunos realizaram as práticas de meditação duas vezes por semana, em um período que variava de 20 a 30 minutos, o que totalizou, aproximadamente, 10 horas de realização de mindfulness. Ao final do estudo, verificou-se que o grupo experimental apresentou ganhos de competência social, além de ganhar notas mais elevadas no boletim em domínios de aprendizagem, saúde e domínio sócio-emocional.

Seguindo uma abordagem exploratória [Wisner 2013], um estudo com 35 adolescentes de um colégio americano, com idade entre 15 e 19 anos, realizou sessões de mindfulness durante o período de oito semanas, com sessões que foram aumentando o tempo de prática durante cada encontro, com tempos progressivos de 4,10 e 30 minutos. Além das sessões no colégio, os estudantes também foram estimulados a meditar em seus lares. Ao fim de cada sessão, os estudantes respondiam a uma questão que buscava entender os benefícios advindos do processo da meditação. Ao final do experimento, realizou-se uma de análise de dados, a qual indicou ganhos em dimensões como a melhora na habilidade de prestar atenção, além da melhora na gestão de estresse e engajamento dos alunos.

Em um estudo experimental [Beauchemin et al. 2008], 34 estudantes diagnosticados com problemas de aprendizagem passaram por sessões semanais de mindfulness, com duração de 45 minutos para as duas sessões iniciais, e com duração de cinco a dez minutos para as cinco sessões semanais seguintes. Através de instrumentos aplicados no início do estudo, verificou-se que estudantes com dificuldades de aprendizagem apresentavam características como altos níveis de ansiedade, maiores traços de estresse e menores habilidades sociais quando comparados a alunos sem dificuldade de aprendizagem. Participaram do estudo estudantes com idades que variavam entre 13 e 18 anos. Foram aplicados instrumentos, tais como um ranking de habilidades sociais, um inventário de ansiedade e um questionário de atitudes, que versava sobre o próprio foco na sala de aula segundo percepção do aluno. Todos os instrumentos foram aplicados antes de iniciar a intervenção e no final (após as sete semanas). Verificaram-se ganhos significativos quanto a traços de ansiedade e estresse, o que indica que a prática de mindfulness pode ser válida para a melhora da 
aprendizagem, uma vez que esses estados afetivos, geralmente, prejudicam a mesma, conforme indica o trabalho.

Em processo similar, um estudo piloto realizado com três alunos estudantes do Ensino Fundamental mostrou que a prática de mindfulness pode ser efetiva para o engajamento dos alunos nas atividades escolares [Felver 2013]. Para tanto, três estudantes com problemas de engajamento e características opositoras à professora participaram de sessões de mindfulness com duração entre 20 e 30 minutos, durante cinco encontros. Os dados foram obtidos por meio de questionários, aplicados aos alunos e suas professoras.

Como se percebe, a área de pesquisa do mindfulness com estudantes tem apresentado resultados efetivos em relação à atenção, além da diminuição de estresse e ansiedade, dimensões que estão interligadas à aprendizagem.

\section{Trabalhos Relacionados: Mindfulness em Ambientes Computacionais}

Embora não tenha sido encontrado o relato de trabalhos que utilizaram o mindfulness em algum STI ou outro ambiente computacional de aprendizagem, inteligente ou não, o campo de computação e meditação tem crescido, demonstrando alguns estudos iniciais. Uma avaliação piloto, com um treinador virtual no formato de agente pedagógico, indicou que o treinamento fornecido pelo personagem foi mais efetivo do que o praticado por usuário em outros meios, como áudio e livros de treinamento [Hudlicka 2011]. Assim sendo, pode-se pensar que um agente pedagógico, que regula os treinamentos, também pode ser útil quando comparado a outros meios da prática de mindfulness.

Outra experiência em que participantes demonstraram benefícios a partir da interação de meditação com um computador foi o sistema denominado "Sonic Cradle". Participantes ficaram suspensos no escuro, deitados em uma suspensão confortável (uma espécie de rede), e a partir da interação com o computador, podiam controlar sons com a própria respiração, o que sugeriu uma experiência similar às outras técnicas de mindfulness [Vidyarthi e Riecke 2014].

Outro campo em que a prática guiada de mindfulness está presente é na aplicação mobile. Um aplicativo chamado NEON foi desenvolvido com o objetivo de promover a meditação no próprio celular [Chittaro e Sioni 2014], a partir da inserção de um áudio que orienta a experiência da prática de mindfulness. Um estudo realizado com o aplicativo em questão, com participantes universitários, demonstrou que o NEON pode ser eficaz quando comparado a outras duas técnicas tradicionais. A avaliação em questão foi realizada com instrumentos qualitativos que avaliaram a experiência dos participantes.

\section{Método}

Para a realização do trabalho, foram selecionadas duas turmas de sétimo ano de um colégio particular da região do Vale dos Sinos, no Rio Grande do Sul, com alunos de idade entre 12 e 14 anos. Inicialmente, os alunos receberam um documento com a explicação das atividades, o qual estava embasado em todos os parâmetros éticos que regem a pesquisa. Em seguida, receberam o TCLE (Termo de Consentimento Livre e Esclarecido), que deveriam retornar assinados caso concordassem com os procedimentos relatados. Como os alunos não possuíam a maioridade, de acordo com a 
legislação brasileira, os responsáveis legais tiveram de assinar o documento, concordando com a participação dos alunos na atividade. O projeto e TCLE foram aprovados pelo Comitê de Ética da Universidade do Vale do Rio dos Sinos. Todos participantes devolveram o documento com a assinatura de seus responsáveis e puderam participar da pesquisa.

Primeiramente, todos os alunos foram agrupados em uma lista geral, que em seguida gerou dois grupos diferentes para a utilização do sistema tutor inteligente, sendo um experimental e outro de controle. Os grupos foram separados aleatoriamente pelo software estatístico SPSS. Em um primeiro momento, 59 alunos foram divididos entre os dois grupos, de modo que o grupo experimental contou com 30 usuários e o de controle com 29 usuários. Os alunos que não realizaram o pré-teste ou o pós-teste foram eliminados da pesquisa, o que gerou, no final, o número de 46 participantes. $\mathrm{O}$ grupo experimental foi formado por 24 alunos e o controle por 22 alunos. Os alunos em questão foram selecionados pelo fato de estarem no processo de aprendizagem de álgebra elementar, e assim utilizaram o sistema tutor inteligente PAT2Math durante quatro encontros, em semanas consecutivas. A Figura 1 ilustra o desenho da avaliação experimental realizada.

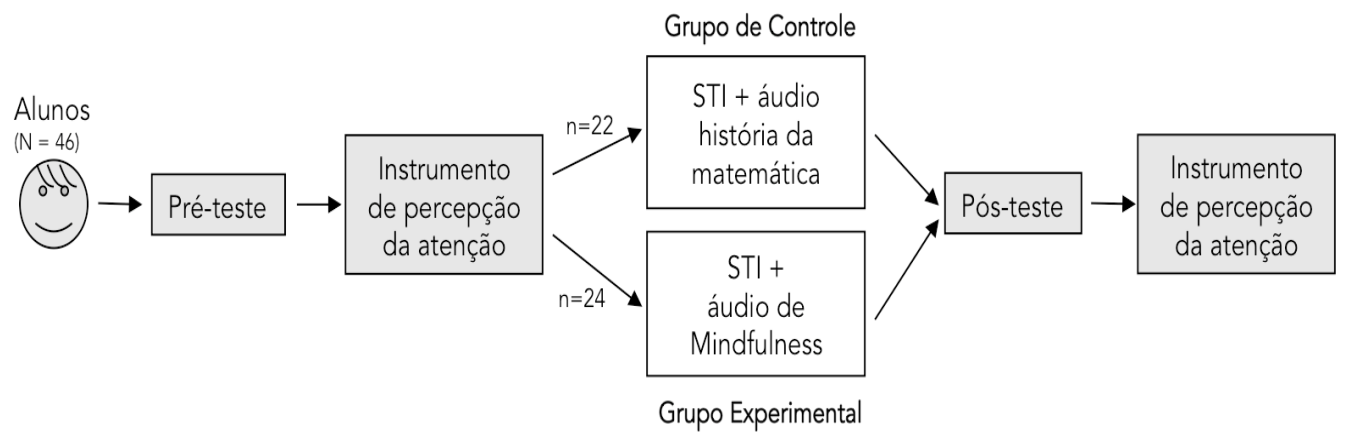

Figura 1. Desenho da avaliação experimental

O grupo experimental teve como diferencial a realização da prática de mindfulness durante o período aproximado de cinco minutos, com o objetivo de melhorar a aprendizagem destes alunos. O grupo de controle, durante o mesmo tempo, ouviu uma narração sobre a origem da álgebra, que serviu como efeito placebo. Assim que o aluno realizava login no sistema, aparecia a imagem de um personagem meditando, enquanto o áudio com a atividade era carregado em uma barra de progresso. Se algum aluno tentasse burlar este procedimento, fechando a página web do STI ou o navegador web, quando acessava novamente o sistema, acontecia o mesmo processo de aparecer o agente e em seguida o áudio, que direcionava para meditação ou mesmo a história sobre a álgebra, dependendo do grupo em que o aluno estava inserido. Ambos os áudios foram gravados por um especialista em locução.

A técnica de mindfulness utilizada foi retirada do livro intitulado "A prática da psicoterapia cognitivo-comportamental baseada em mindfulness e aceitação" [Roemer e Orsillo 2010], utilizada em intervenções clínicas e cientificamente validada. Assim, utilizou-se um conjunto de passos que foram gravados pelo locutor, de modo pausado e lento, para a posterior prática dos estudantes que compuseram o grupo de experimento. Os passos, repetindo-se em um espaço de tempo, eram: "Fique em uma posição confortável na cadeira. Fique com a coluna ereta, mas não rígida. Relaxe o seu corpo e 
deixe os ombros caírem, naturalmente. Feche os seus olhos e não se preocupe com outras questões, mantenha sua concentração apenas na tarefa. Se surgirem outros pensamentos, apenas observe. Respire! Puxe e solte o ar... Preste atenção nos movimentos de sua barriga e no ar entrando e saindo de suas narinas, lentamente. $\mathrm{O}$ ar entrando e saindo é como uma onda, preste atenção nas sensações de sua narina com o movimento do ar. Pensamentos podem surgir em sua mente, ela tenta fazer você pensar em outras situações... Apenas observe os pensamentos e não julgue e nem se preocupe com eles. Suavemente, volte sua atenção para a sua respiração. Preste atenção de novo em sua barriga, enquanto respira. Os movimentos de ida e vinda, bem vagarosamente. A sua mente pode trazer mil outros pensamentos, apenas observe. Continue prestando atenção na sua respiração".

Em relação ao áudio do grupo controle, foram gravadas três narrações acerca da história da álgebra, que versavam acerca de algum acontecimento histórico deste campo. Esse áudio também foi gravado de modo pausado e lento, igual à técnica de mindfulness, e com os mesmos cinco minutos. Como exemplo deste procedimento, um trecho de uma das histórias é: " $\mathrm{Na}$ Índia antiga, há muito tempo, a Matemática era muito difícil. Sem nenhum sinal, sem nenhuma variável, somente alguns poucos sábios eram capazes de resolver os problemas, usando muitos artifícios e trabalhosas construções geométricas. Hoje, temos a linguagem exata para representar qualquer quebra-cabeça ou problema; basta traduzi-los para o idioma da Álgebra: a equação....”.

Para a avaliação da influência do mindfulness na aprendizagem, utilizou-se um pré e pós-teste em formato de prova, com equações de primeiro grau. Em relação à atenção, os alunos de Psicologia, orientados por um psicólogo especialista em avaliação psicológica, desenvolveram uma escala de percepção de atenção. $\mathrm{O}$ documento em questão contava com dezesseis comportamentos relativos a alunos atentos, em uma escala de 0 a 10. Assim sendo, a professora de matemática deveria pontuar as ações de cada aluno no início e fim do experimento. Com o objetivo de preservar a neutralidade do experimento, em nenhum momento foi relatado à professora que a pesquisa em questão visava verificar o ganho de atenção e aprendizagem dos alunos participantes.

\section{Materiais}

A escala foi construída a partir da orientação de um psicólogo responsável, especialista no tema de avaliação psicológica, utilizando como referência as contribuições de Pasquali (1999). Primeiramente, uma entrevista com uma professora de matemática foi realizada, com uma pergunta aberta questionando o que era um aluno atento (construto psicológico da atenção) e seus comportamentos específicos. A partir das respostas fornecidas, foi elaborada uma escala piloto, contendo os comportamentos (itens) relativos à atenção, que depois sofreram correções. O processo de construção da escala é ilustrado na Figura 2.

Os autores identificaram dezesseis categorias contendo atitudes e comportamentos de um aluno atento. Cada item da escala poderia ser pontuado de 0 a 10, conforme escala Likert, tendo alguns itens com sentido inverso. Na maioria dos comportamentos, a pontuação de uma atitude de atenção tendia a 10, como "Interage com o professor", com algumas exceções que tendiam a 0 , como "necessita que chame a atenção". A escala não foi desenvolvida com o intuito de medir a atenção, mas sim a percepção da atenção do aluno pela professora. 
A medida estatística que indica a capacidade de uma escala medir o que propõe se chama Alfa de Cronbach. A análise posterior dos instrumentos, após a resposta da professora, indicou um índice de confiabilidade de 0,906, o que indica que a escala consegue mensurar o que se propõe.

O pré-teste e pós-teste de álgebra contou com cinco questões, totalizando dez exercícios. Ambos os testes foram construídos com questões com nível crescente de complexidade. Cada questão testava o conhecimento do aluno em diferentes operações matemáticas e eram estruturalmente similares às equações apresentadas para o aluno resolver no STI PAT2Math. No total, quatro diferentes provas foram desenvolvidas, todas similares em relação ao formato. Utilizaram-se duas provas de álgebra similares no pré-teste (uma para cada grupo) e outras duas similares no pós-teste.

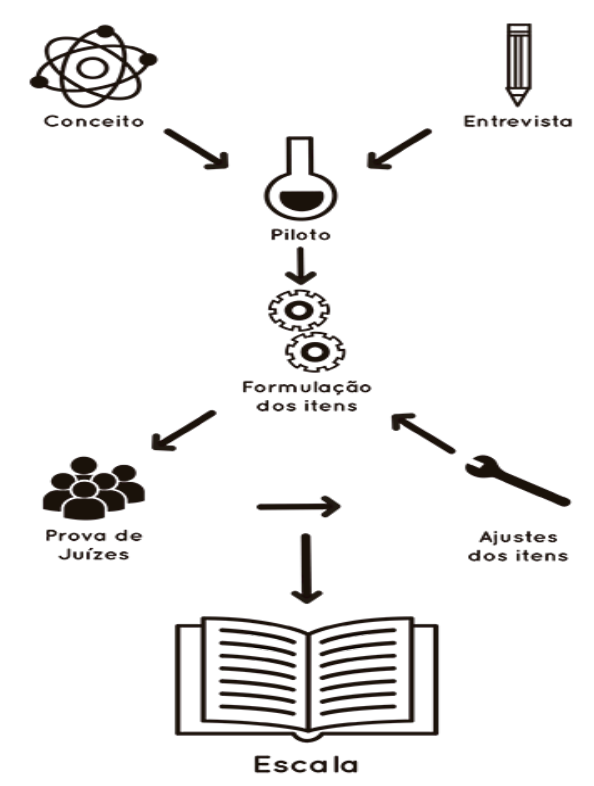

Figura 2. Modelo de construção da escala de atenção

\section{Análise e Discussão dos Resultados}

Este estudo piloto visou identificar a possível melhora na atenção e, consequentemente, aprendizagem de estudantes que utilizaram um STI para resolver equações algébricas, depois de realizarem a prática de mindfulness neste ambiente virtual de aprendizagem. A partir da utilização de uma escala de percepção da atenção e uma prova de álgebra, ambos em caráter de pré-teste e pós-teste, os dados passaram por análises estatísticas.

Para verificar se existiu ganho de aprendizagem, foram comparadas as médias dos pós-testes dos grupos de controle e experimental. Foi utilizado o Teste T de Student para amostras independentes visando verificar se o ganho ${ }^{2}$ de nota do grupo experimental havia sido significativamente maior quando comparado ao controle. $\mathrm{O}$ resultado estatístico, no entanto, não indicou um valor significativamente estatístico ( $\mathrm{p}>$ $0,05)$. Assim sendo, não há indícios significativos de que a prática de mindfulness interferiu na melhora de desempenho dos estudantes. Ambos os grupos apresentaram

\footnotetext{
${ }^{2} \mathrm{O}$ ganho é a diferença entre a nota do pós-teste e a nota do pré-teste para cada aluno.
} 
resultados superiores no pós-teste em relação ao pré-teste, o que não assegura a eficácia do mindfulness e sugere um provável resultado ao fato de os usuários terem aprendido pelo uso do sistema tutor inteligente.

Em relação à percepção da atenção, realizando o teste estatístico ANOVA (Análise Univariada de Variância) de medidas repetidas, foi verificado que alguns itens relacionados à atenção tiveram diferença estatística durante o experimento $(p<0,05)$. Os comportamentos de interagir com o professor, responder quando solicitado, manter o contato visual e manter o foco no professor durante a explicação tiveram resultados significativos em ambos grupos quando realizada a comparação por meio da ANOVA, conforme demonstra a tabela 1. Embora a variável que diferencia os dois grupos seja o mindfulness, pode-se atentar para outra similaridade que esteve presente em ambos os grupos, que é o direcionamento da atenção dos participantes para um estímulo específico, o que envolve a prática da concentração e pode ter sido decisivo na mudança de alguns itens relacionados à atenção e, eventualmente, no resultados do pós-teste. Deste modo, acredita-se que o ato de ouvir um áudio sobre a história da matemática para os estudantes do grupo de controle, concentrando-se na mesma, pode ter sido uma experiência similar à prática de mindfulness. Isso pode ser uma possível explicação para que fosse observada uma melhora em ambos os grupos: controle e experimental.

Tabela 1. Comparação das questões da escala de atenção em duas situações: (1) antes e após o experimento; (2) grupo de controle versus grupo experimental

\begin{tabular}{|l|c|c|}
\hline \multicolumn{1}{|c|}{ Questão } & $\begin{array}{c}\text { Antes X } \\
\text { Após }\end{array}$ & $\begin{array}{c}\text { Experimento } \\
\text { X Controle }\end{array}$ \\
\hline 1. Faz perguntas sobre a matéria & N.S* & N.S* \\
\hline 2. Se dispersa com facilidade & N.S* & N.S* \\
\hline 3. Ouve em silêncio & N.S* & N.S* \\
\hline 4. Interage com o professor & $\mathbf{p}<\mathbf{0 , 0 5 0}$ & N.S* \\
\hline 5. Necessita que chame a atenção & N.S* & N.S* \\
\hline 6. Faz os exercícios & N.S* & N.S* \\
\hline 7. Responde quando solicitado & $\mathbf{p}<\mathbf{0 , 0 5 0}$ & N.S* \\
\hline 8. Esquece o tema de casa & N.S* & N.S* \\
\hline 9. Entende a matéria com facilidade & N.S* & N.S* \\
\hline $\begin{array}{l}\text { 10. Tem bom desempenho na } \\
\text { disciplina }\end{array}$ & N.S* & N.S* \\
\hline 11. Acerta todo o exercício & N.S* & N.S* \\
\hline 12. Relata dúvida & N.S* & N.S* \\
\hline 13. Mantém contato visual & $\mathbf{p}<\mathbf{0 , 0 0 1}$ & N.S* \\
\hline $\begin{array}{l}\text { 14. Mantém o foco no professor } \\
\text { durante a explicação }\end{array}$ & $\mathbf{p}<\mathbf{0 , 0 5 0}$ & N.S* \\
\hline 15. Comete erros nos exercícios & N.S* & N.S* \\
\hline 16. É atento & N.S* & N.S* \\
\hline
\end{tabular}

N.S* Não significativo

A Tabela 1 indica os resultados encontrados a partir do ANOVA em relação às mudanças de medidas de todos os participantes antes e após o experimento (considerando os dados de ambos os grupos). A coluna da direita analisou a variância em cada grupo no início e fim do experimento, com o objetivo de verificar se houve alguma mudança significativamente estatística do grupo experimental em relação ao de controle. 


\section{Conclusão e Trabalhos Futuros}

O presente artigo descreveu um estudo piloto que visou verificar a influência da prática de mindfulness na melhora da atenção, e por consequência, aprendizagem algébrica de estudantes que se submeteram a práticas de meditação em um sistema tutor inteligente. No entanto, através da aplicação do teste estatístico T de student, não foram encontradas evidências significativas que a prática de mindfulness possa ter levado a uma melhor aprendizagem com o auxílio do STI.

Contudo, quatro alterações em comportamentos relativos à atenção, que aconteceram nos dois grupos, fornecem indício que a similaridade das experiências pode favorecer a melhora em alguns aspectos. Por se tratar de um estudo piloto, os achados em questão são interessantes e sugerem o interesse de uma continuação do trabalho, com algumas melhoras metodológicas.

Ainda são poucos e preliminares os estudos que envolvem a prática de mindfulness em ambientes computacionais. No caso em específico de sistemas tutores inteligentes ou até mesmos ambientes computacionais de aprendizagem não inteligentes, não foi encontrado nenhum trabalho que indica a tentativa de agrupar os dois campos em questão. Assim sendo, se pensa que o trabalho desenvolvido faz parte de uma tentativa inovadora de juntar duas áreas de resultados significativos no campo científico, que são os sistemas tutores inteligentes e o mindfulness.

O trabalho em questão continuará a ser desenvolvido, com novos experimentos que visam a correção de elementos que possam ter sido entraves na obtenção de um resultado positivo. A busca de produções científicas que utilizaram o mindfulness na melhora da aprendizagem e atenção indicaram trabalhos com uma maior margem de tempo para o desenvolvimento da técnica de meditação, bem como uma maior quantidade de encontros [Flook et al. 2015; Wisner 2013]. Outro ponto focal das avaliações futuras é a necessidade de utilizar outro instrumento capaz de verificar o ganho de atenção imediatamente após o usuário ter realizado a prática de mindfulness, além da escala de percepção de atenção já desenvolvida e utilizada.

\section{Agradecimentos}

O presente trabalho foi realizado com o apoio dos seguintes órgãos de fomento à pesquisa: CNPq e FAPERGS.

\section{Referências}

Baer, R. A. (2003) Mindfulness training as a clinical intervention: A conceptual and empirical review. Clinical Psychology: Science and Practice, 10, 125-143.

Beauchemin, J.; Hutchins, T.; Patterson, F. (2008) Mindfulness Meditation May Lessen Anxiety, Promote Social Skills, and Improve Academic Performance Among Adolescents With Learning Disabilities. Complementary Health Practice Review, v. 13, n. 1, p. 34-45.

Chittaro, L.; Vianello, A. (2014) Computer-supported mindfulness: Evaluation of a mobile thought distancing application on naive meditators. International Journal of Human-Computer Studies, 72(3), 337-348. 
Davidson, R.; Lutz, A. (2008) Buddha's brain: neuroplasticity and meditation. IEEE Signal Process Mag. 25(1): 176-174.

Felver, J.; Frank, J.; McEachern, A. (2013) Effectiveness, Acceptability, and Feasibility of the Soles of the Feet Mindfulness-Based Intervention with Elementary School Students. Mindfulness, v. 5, n. 5, p. 589-597.

Flook, L. et al (2015) Promoting prosocial behavior and self-regulatory skills in preschool children through a mindfulness-based kindness curriculum. Developmental Psychology, v. 51, n. 1, p. 44-51.

Hudlicka, E. (2011) Virtual Coach for Mindfulness Meditation Training, 17-24.

Kabat-Zinn, J. (1990) Full catastrophe living: Using the wisdom of your body and mind to face stress, pain and illness. New York: Delacorte.

Ma, W.; Adesope, O. O.; Nesbit, J. C.; Liu, Q. (2014) Intelligent Tutoring Systems and Learning Outcomes: A Meta-Analysis. Journal of Educational Psychology, v. 106, n. 4, p. 1-18.

Oord, S. van der; Bögels, S. M.; Peijnenburg, D. (2012) The Effectiveness of Mindfulness Training for Children with ADHD and Mindful Parenting for their Parents. Journal of Child and Family Studies, 21(1), 139-147.

Pasquali, L. (1999) Instrumentos psicológicos: guia prático de elaboração. Brasília: Ibapp.

Paula, G. R. et al (2006) Neuropsicologia da aprendizagem. Rev. psicopedag., São Paulo, v. 23, n. 72.

Roemer, L.; Orsillo, S. M. (2010) A prática da terapia cognitivo-comportamental baseada em mindfulness e aceitação. Porto Alegre: Artmed.

Steenbergen-Hu, S.; Cooper, H. (2014) A meta-analysis of the effectiveness of intelligent tutoring systems on college students' academic learning. Journal of Educational Psychology, v. 106, p. 331-347.

Steenbergen-Hu, S.; Cooper, H. (2013) A meta-analysis of the effectiveness of intelligent tutoring systems on $\mathrm{K}-12$ students' mathematical learning. Journal of Educational Psychology, v. 105, p. 970-987.

Vanlehn, K. (2011) The Relative Effectiveness of Human Tutoring, Intelligent Tutoring Systems, and Other Tutoring Systems. Educational Psychologist, v.46, n.4, p. 197221.

Vidyarthi, J.; Riecke, B. (2014) Interactively mediating experiences of mindfulness meditation. Internat. Journal of Human-Computer Studies, v. 72, n. 8-9, p. 674-688.

Weare, K. (2012) Evidence for the Impact of Mindfulness on Children and Young People. The Mindfulness In Schools Project, 1-12.

Wisner, B. (2013) An Exploratory Study of Mindfulness Meditation for Alternative School Students: Perceived Benefits for Improving School Climate and Student Functioning. Mindfulness, v. 5, n. 6, p. 626-638.

Woolf, B. P. (2010) Building intelligent interactive tutors: Student-centered strategies for revolutionizing e-learning. Morgan Kaufmann. 